\title{
Effect of Kinetic Parameters on I-129 Activity from Fuel to Coolant in PWRs
}

\author{
Rubina Nasir \\ Department of Physics, Air University, Islamabad, Pakistan \\ Email: rubinanm@yahoo.com,rubina.nasir@mail.au.edu.pk
}

How to cite this paper: Nasir, R. (2017) Effect of Kinetic Parameters on I-129 Activity from Fuel to Coolant in PWRs. World Journal of Nuclear Science and Technology, 7, 284-291. https://doi.org/10.4236/wjnst.2017.74022

Received: August 28, 2017

Accepted: October 16, 2017

Published: October 19, 2017

Copyright (c) 2017 by author and Scientific Research Publishing Inc. This work is licensed under the Creative Commons Attribution International License (CC BY 4.0).

http://creativecommons.org/licenses/by/4.0/

\section{(c) (i) Open Access}

\begin{abstract}
Effect of kinetic model parameters on fission product (I-129) activity from fuel to coolant in PWRs has been studied in this work. First a computational model was developed for fission product release into primary coolant using ORIGEN-2 as subroutine. The model is based on set of differential equations of kinetic model which includes fuel-to-gap release model, gap-to-coolant leakage model, and Booths diffusion model. A Matlab based computer program FPAPC (Fission Product Activity in Primary Coolant) was developed. Variations of I-129 activity in Primary Heat Transport System were computed and computed values of i-129 were found in good agreement and deviations were within $2 \%-3 \%$ of already published data values. Finally, the effects of coolant purification rate, diffusion constant and gas escape rate on I-129 activity were studied and results indicated that the coolant purification rate is the most sensitive parameter for fission product activity in primary circuit. For changes of $5 \%$ in steps from $-10 \%$ to $+10 \%$ in the coolant purification rate constant $(\beta)$, the activity variation after 200 days of reactor operation was $23.1 \%$ for the change.
\end{abstract}

\section{Keywords}

Kinetic Model, Fission Product Activity, Coolant Purification Rate, Diffusion Constant, Gas Escape Rate, PWRs, I-129

\section{Introduction}

Majority of world operating reactors and systems in planning are Pressurized Water Reactors (PWR) or advanced PWR type [1] [2]. Due to their high capacity factors, these reactors are used as baseline energy producing units. During full power operation, the primary coolant loops of PWR systems have relatively high levels of radiation doses [3] [4]. 
The fission product activity (FPA) is one of the important contributors to the total doses near primary coolant circuit. These levels are continuously monitored during the normal operation of PWRs [5]. The fuel pins develop leakages with their burnup. When the failed fuel fraction exceeds a safe limit, replacement of defective assemblies becomes necessary. The total fission product activity can become available source to escape into the environment [6] [7] [8]. An analytic procedure to estimate the fission products leakage into the gap was suggested in past [8]. In this method, it was assumed that the radionuclides can escape from the grain corners and leak into the gap. Then Lewis gave a model for diffusion of fission fragments in fuel and then leaked into the gap and finally transferred to coolant through cracks of cladding [9]. It also included the effect of coolant purification rate, diffusion constant and gas escape rate that strongly affect the FPA in the system. Also recently, a detailed overview has been presented by Lewis et al. for fission product release modelling for application of fuel-failure monitoring and detection [10].

When a fuel becomes defective, the coolant can enter into the gap via cladding and fission fragments (i.e., notably the volatile species of noble gas and iodine) can escape. Then the fuel integrity can get compromised and it will enhance the fission product release. The thermal conductivity of the fuel decreases and heat transfer becomes poor when the system is allowed to operate under defected conditions. Therefore, defective fuel bundles must be discharged from the core as soon as possible.

This work aims at first developing a time-dependent mathematical model for a typical fission product release from the fuel matrix, into fuel-to-clad gap and from gap into the primary coolant. The isotope I-129 was selected as a typical fission product. As a first step, the fission product release into fuel is done by using ORIGEN-2 [11] as subroutine. Then the Booths diffusion model is used for fission product transfer from fuel matrix into the fuel-to-clad gap. Furthermore, the balance equations that govern the fission product inventory in the gap are used to transport some fraction into the primary coolant via leakages from cladding. Then the fission product activity in the coolant is estimated and compared with the published data. Finally, the effect of coolant purification rate, diffusion constant and gas escape rate on fission product activity in the system was determined.

\section{Method}

\subsection{Source Term}

The amount of radioactive material released in an accident is called the source term or the measure of radioactive contamination. It is directly proportional to the amount of core inventory and is a function of core fissile content, reactor power, fuel burn up, neutron flux distribution, and operating history among others. The characteristics of radionuclides also influence the source term. The nuclides include noble gases (Xe, $\mathrm{Kr}$ ), volatile isotopes (I, Cs, Te), and semi vola- 
tile isotopes ( $\mathrm{Sr}, \mathrm{Ba}, \mathrm{Ru})$. The isotope I-129 was selected as a typical fission product in this work for which experimental data was available [9]. Its release into the fuel pellet is computed by using ORIGEN-2 computer program [10] as subroutine.

\subsection{Methodologies Adopted}

Based on initial work by Lewis et al. [9], the fission product coolant activity of I-129 was estimated using diffusion model coupled with a mass balance in the gap and coolant. Detailed overview of fission product release modelling can be seen in recent work by Lewis et al., for the application of fuel-failure monitoring and detection [10]. This model is used to predict the coolant activity behavior for both steady-state and transient reactor operations. The model employs variable reactor power and coolant purification histories. It can be matched to coolant activity trends for practical cases and can be used to predict the coolant activity as a function of reactor power and the coolant purification history. Boothdiffusion model for an idealized fuel grain sphere of radius a is used for the radial diffusion equation for the concentration $C(r, t)$ at time with $D(t)$ is diffusion coefficient, $F_{f}$ as fission rate and $V$ as active volume:

$$
\frac{\partial C(r, t)}{\partial t}=\frac{D(t)}{r^{2}} \frac{\partial\left(r^{2} \frac{\partial C(r, t)}{\partial r}\right)}{\partial r}-\lambda C(r, t)+\frac{F_{f}(t) y}{V}
$$

Now multiply above equation by total volume of defective fuel $(V)$ and normalized equation is obtained:

$$
\frac{\partial u(\eta, t)}{\partial t}=\frac{D^{\prime}(t)}{\eta^{2}} \frac{\partial\left(\eta^{2} \frac{\partial u(\eta, t)}{\partial \eta}\right)}{\partial \eta}-\lambda u(\eta, t)+F_{f}(t) y
$$

where

$$
\begin{aligned}
& u(\eta, t)=C(r, t) * V \\
& D^{\prime}(t)=D(t) / a
\end{aligned}
$$

The initial and boundary conditions are:

$$
\begin{aligned}
& \frac{\partial u}{\partial t}=0 ; \eta=0, \quad t>0 . \\
& u(1, t)=0, \quad \eta=1, \quad t>0 .
\end{aligned}
$$

The normalized concentration at start of reactor $(t=0)$ is zero. The rate of diffusion is then given by

$$
R_{d i f}=-3 D^{\prime} \frac{\partial u}{\partial t}
$$

The mass balance in fuel-clad gap is given by the following differential equation.

$$
\frac{\mathrm{d} N_{g}(t)}{\mathrm{d} t}=R_{d i f}(t)-\{\lambda+v(t)\} N_{g}(t)
$$


The concentration in fuel-clad is zero at time $t=0$.

The mass balance of fission products in primary heat transport system (PHTS) is given by

$$
\frac{\mathrm{d} N_{c}(t)}{\mathrm{d} t}=v(t) N_{g}(t)-\{\lambda+\beta(t)\} N_{c}(t)
$$

The concentration in primary coolant circuit initially (at $t=0$ ) is zero. Here, $\beta$ is coolant purification rate; $D^{\prime}$ is Empirical Diffusion Constant; $F_{f}$ is the fission rate or knowledge of core inventory based on ORIGEN code and $v$ is gas escape rate coefficient. Using above Equations, a Matlab based computer program FPAPC (Fission Product Activity in Primary Coolant) was developed. We first have used the approach adopted by Lewis and then incorporated detailed isotope balance in primary coolant circuit as well.

\section{Results and Discussion}

The schematic diagram of the fission product production and removal mechanisms is shown as Figure 1. The fission products are released into the fuel pellet

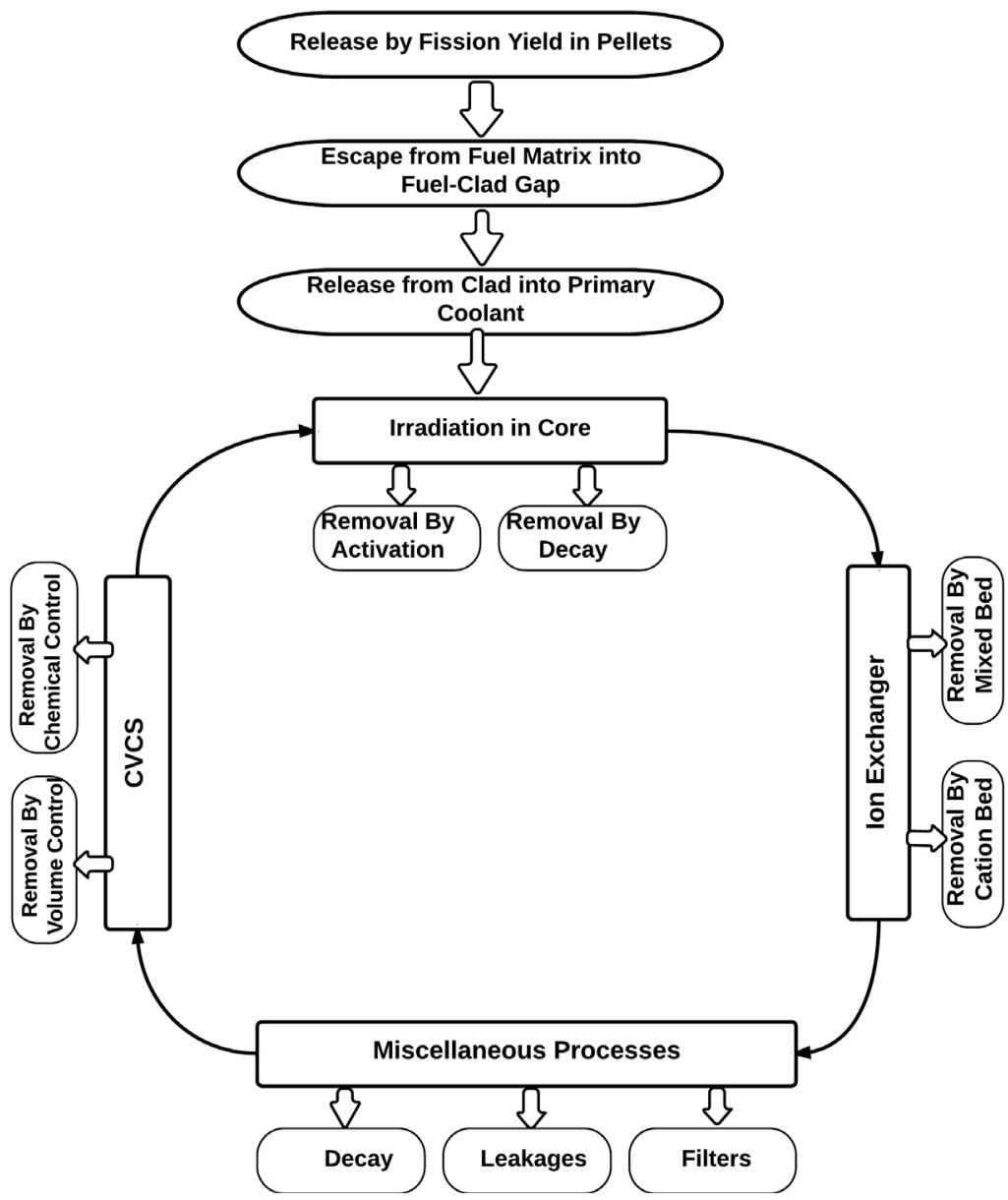

Figure 1. Schematic diagram of the fission products production and removal mechanism in the primary circuit of a typical PWR. 
and are estimated using ORIGEN code. Then the fraction of I-129 that gets escaped into the fuel-clad gap is estimated using diffusion model equations (Equation (1)-(5)). The release from clad to the primary coolant is computed using diffusion and leakage model equations (Equations (5) \& (6)). Then the coolant and its impurities get irradiated in the core and decay of radioactive isotopes also occurs. Moreover, nuclear reactor has the volume control system and the chemical control systems to remove fission fragments along with ion-exchangers to remove activity by cation and mixed beds respectively. Also, some fraction of coolant gets filtered and leaked before the coolant gets back to the core again.

Results for I-129 activity in the primary coolant are shown as Figure 2. For reactor's normal operations the coolant purification rate constant is $7.05 \mathrm{E}-5 \mathrm{~s}^{-1}$; the empirical diffusion coefficient, $D^{\prime}$ is $4.57 \mathrm{E}-10 \mathrm{~s}^{-1}$ and gas escape rate coefficient $(v)$ is $1.4 \mathrm{E}-6 \mathrm{~s}^{-1}$ respectively. Results show that for normal operation the I-129 activity is very close to the values reported by Lewis et al. [9] and remained within $2 \%-3 \%$.

As a next step, we have changed the coolant purification rate constant and studied its effect on the activity. Figure 2 shows that for changes from $-10 \%$ to $+10 \%$ in the coolant purification rate constant $(\beta)$, the normalized activity after 200 days of operation changed from 2.6 to 3.2 and it indicates that system is sensitive to changes in coolant purification rate constant. For changes of $5 \%$ in steps from $-10 \%$ to $+10 \%$ in the coolant purification rate constant $(\beta)$, the activity variation after 200 days of operation was $23.1 \%$ for the change and it indicated that system is quite sensitive to the changes in coolant purification rate constant.

Similarly, when diffusion constant (D') was allowed to change keeping others parameters fixed, the results for I-129 activity in the primary coolant are shown

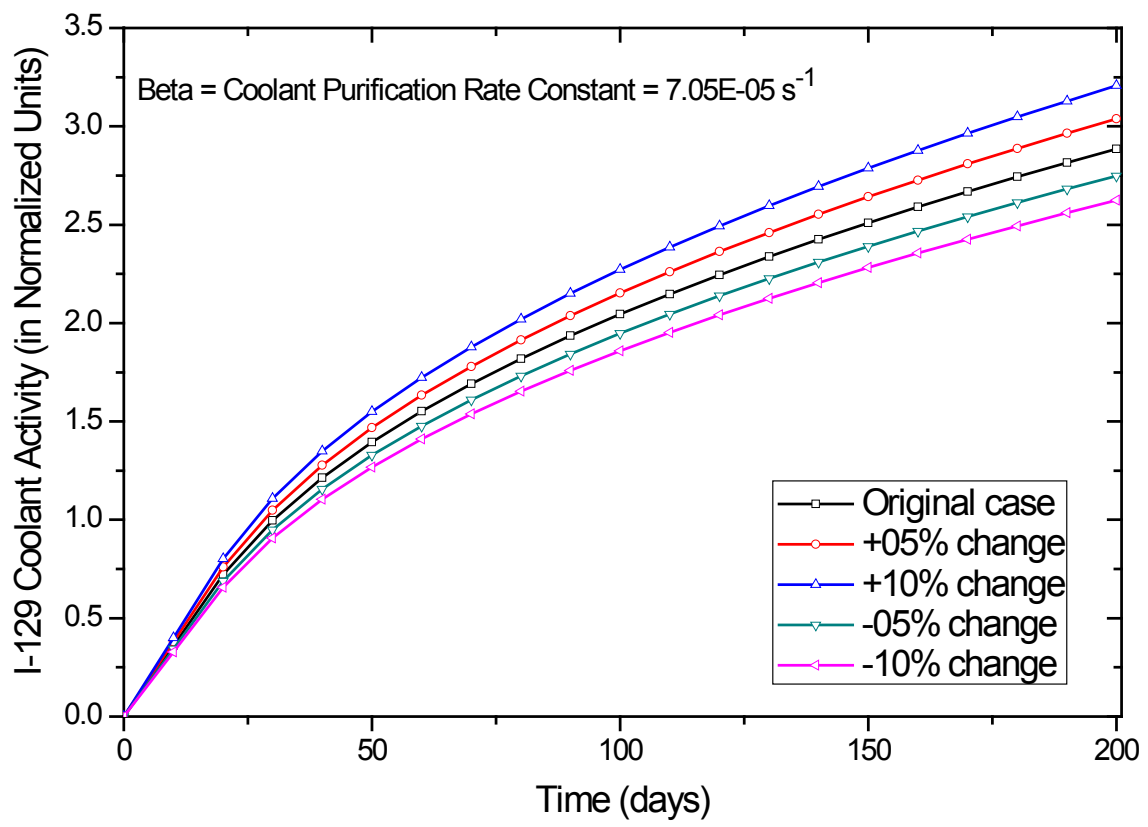

Figure 2. Effect of coolant purification rate $(\beta)$ on normalized I-129 coolant activity for small perturbations. Normal operation value of $\beta=7.0 \mathrm{E}-05 \mathrm{~s}^{-1}$. 
as Figure 3. It shows that for changes from $-10 \%$ to $+10 \%$ in the diffusion constant, the activity after 200 days of operation changed only to $9.6 \%$ (i.e. from 2.75 to 3.046$)$ and it indicates that system is not so sensitive to changes in the diffusion constant. Also, when we changed the gas escape rate coefficient $(v)$ from $-10 \%$ to $+10 \%$ of its normal value activity after 200 days of operation changed only to $0.4 \%$. The results for the effect of changes in the gas escape rate coefficient $(v)$ on I-129 activity in primary coolant are shown as Figure 4. It

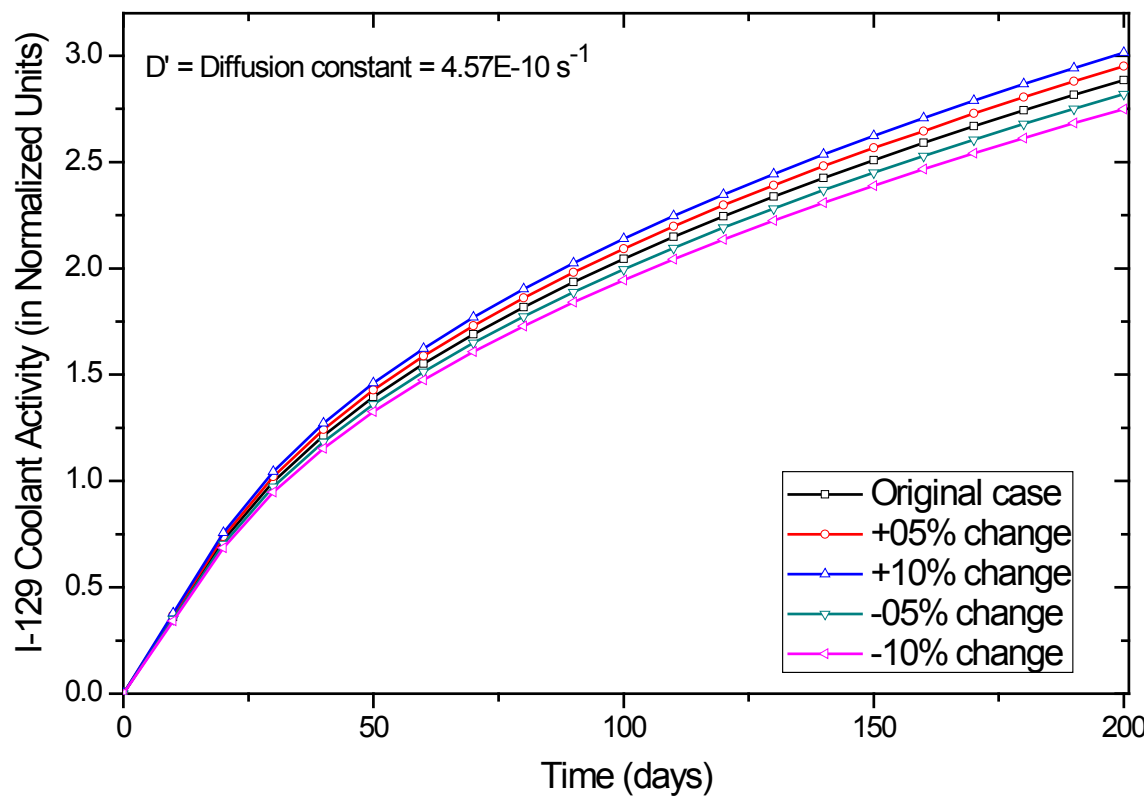

Figure 3. Effect of Diffusion coefficient (D') on normalized I-129 coolant activity for small perturbations. Normal operation value of $\mathrm{D}^{\prime}=4.57 \mathrm{E}-10 \mathrm{~s}^{-1}$.

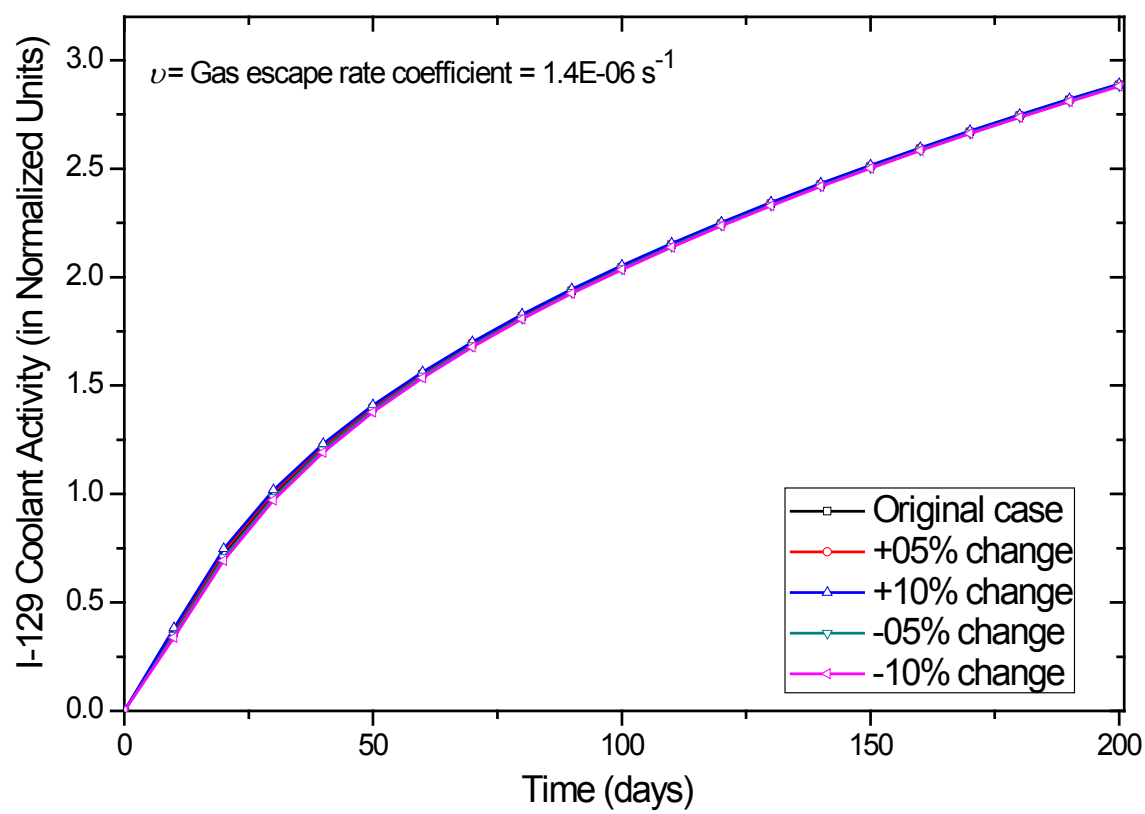

Figure 4. Effect of gas escape rate coefficient $(v)$ on normalized I-129 coolant activity for small perturbations. Normal operation value of $v=1.4 \mathrm{E}-06 \mathrm{~s}^{-1}$. 
indicates that system is not sensitive to changes in gas escape rate coefficient $(v)$ in given domain.

\section{Conclusions}

Effect of kinetic model parameters on fission product (I-129) activity from fuel to coolant in PWRs has been studied in this work. First a computational model was developed for fission product release into primary coolant which uses ORIGEN-2 as subroutine. Variations of I-129 activity in Primary Heat Transport System were computed and were within $2 \%-3 \%$ of already published data values. For changes of $5 \%$ in steps from $-10 \%$ to $+10 \%$ in the coolant purification rate constant $(\beta)$, the activity variation after 200 days of reactor operation was $23.1 \%$ for the change. It shows that the system is quite sensitive to the changes in coolant purification rate constant.

Similarly, when diffusion constant (D') was allowed to shift from $-10 \%$ to $+10 \%$ of its normal value, the I- 129 activity coolant changed only to $9.6 \%$, so system is not so sensitive to these changes. Also, when we perturb the gas escape rate coefficient $(v)$ from $-10 \%$ to $+10 \%$ of its normal value, the activity changed only to $0.4 \%$ showing that the system is not sensitive to such changes.

\section{References}

[1] Klevinskas, G. (2007) Analysis of Iodine Release from the Defective Fuel Elements of the RBMK-1500 Reactor. Lithuanian Journal of Physics, 47, 211-219. https://doi.org/10.3952/lithjphys.47208

[2] Brent, A.H. and Lewis, J. (2003) Modelling the Activity of ${ }^{129} \mathrm{I}$ in the Primary Coolant of PWRs. Journal of Nuclear Materials, 312, 81-96.

[3] Hwang, K.H. (2005) Modeling the Activity and Scaling Factor of 129I in the Primary Coolant and CVCS Resin. Annals of Nuclear Energy, 32, 1898-1917. https://doi.org/10.1016/j.anucene.2005.06.008

[4] Wood, C.J. (1987) Recent Developments in LWR Radiation Field Control. Progress in Nuclear Energy, 19, 241-266. https://doi.org/10.1016/0149-1970(87)90021-7

[5] Comley, G.C. (1985) The Significance of Corrosion Products in Water Reactor Coolant Circuits. Progress in Nuclear Energy, 16, 41-72. https://doi.org/10.1016/0149-1970(85)90005-8

[6] Huang, G.F. (2010) Study on Mitigation of In-Vessel Release of Fission Products in Severe Accidents of PWR. Nuclear Engineering and Design, 240, 3888-3897. https://doi.org/10.1016/j.nucengdes.2010.08.010

[7] Pontillon, Y. (2010) Behaviour of Fission Products under Severe PWR Accident Conditions VERCORS Experimental Programme-Part 1: General Description of the Programme. Nuclear Engineering and Design, 240, 1843-1852. https://doi.org/10.1016/j.nucengdes.2009.06.028

[8] Hyun, K.Y. (1994) An Analysis Method for the Rod Gap Inventory of Unstable Fission Products during Steady-State Operation. Journal of Nuclear Materials, 209, 62-78. https://doi.org/10.1016/0022-3115(94)90248-8

[9] Lewis, B.J. (1990) A Generalized Model for Fission Product Transport in the Fuel-to-Sheath Gap of Defective Fuel Elements. Journal of Nuclear Materials, 175, 218-226. https://doi.org/10.1016/0022-3115(90)90210-E 
[10] Lewis, B.J., Chan, P.K., El-Jaby, A., et al. (2017) Fission Product Release Modelling for Application of Fuel-Failure Monitoring and Detection-An Overview. Journal of Nuclear Materials, 489, 64-83.

[11] Croft, A.G. (1980) A User's Manual for the ORIGEN2 Computer Code. Rep. ORNL/TM-7175, Oak Ridge National Laboratory, Oak Ridge, TN. 